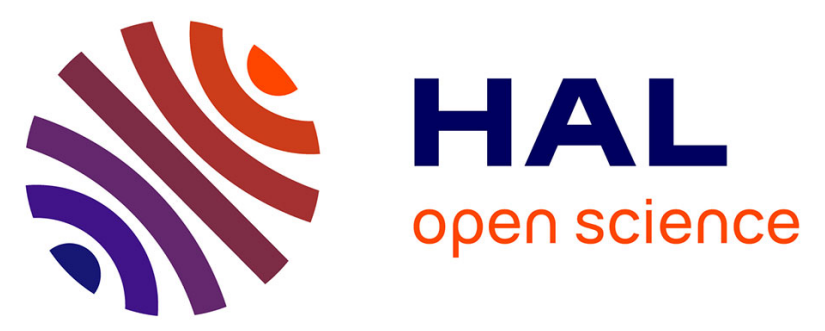

\title{
A crystallographic dislocation model for describing hardening of polycrystals during strain path changes. Application to low carbon steels
}

K. Kitayama, C.N. Tome, E.F. Rauch, J.J. Gracio, F. Barlat

\section{- To cite this version:}

K. Kitayama, C.N. Tome, E.F. Rauch, J.J. Gracio, F. Barlat. A crystallographic dislocation model for describing hardening of polycrystals during strain path changes. Application to low carbon steels. International Journal of Plasticity, 2013, 46, pp.54-69. 10.1016/j.ijplas.2012.09.004 . hal-00850219

\author{
HAL Id: hal-00850219 \\ https://hal.science/hal-00850219
}

Submitted on 21 Apr 2020

HAL is a multi-disciplinary open access archive for the deposit and dissemination of scientific research documents, whether they are published or not. The documents may come from teaching and research institutions in France or abroad, or from public or private research centers.
L'archive ouverte pluridisciplinaire HAL, est destinée au dépôt et à la diffusion de documents scientifiques de niveau recherche, publiés ou non, émanant des établissements d'enseignement et de recherche français ou étrangers, des laboratoires publics ou privés. 


\title{
A crystallographic dislocation model for describing hardening of polycrystals during strain path changes. Application to low carbon steels
}

\author{
K. Kitayama ${ }^{a}$, C.N. Tomé ${ }^{b}$, E.F. Rauch ${ }^{\text {c }}$ J.J. Gracio ${ }^{\mathrm{a}, *}$, F. Barlat ${ }^{\mathrm{d}, \mathrm{a}}$ \\ ${ }^{a}$ Center for Mechanical Technology and Automation, Department of Mechanical Engineering, University of Aveiro, 3810-193 Aveiro, Portugal \\ ${ }^{\mathrm{b}}$ MST Division, Los Alamos National Laboratory, Los Alamos, NM 87545, USA \\ ' Science et Ingénierie des Matériaux et des Procédés - (CNRS UMR 5266) INPG-UJF, BP 46, 38402 Saint Martin d'Hères cedex, France \\ d Materials Mechanics Laboratory, Graduate Institute of Ferrous Technology, Pohang University of Science and Technology, San31, Hyoja-Dong, Nam-Ku, \\ Pohang 790-784, South Korea
}

Polycrystal aggregates subjected to plastic forming exhibit large changes in the yield stress and extended transients in the flow stress following strain path changes. Since these effects are related to the rearrangement of the dislocation structure induced during previous loading, here we propose a crystallographically-based dislocation hardening model for capturing such behavior. The model is implemented in the polycrystal code VPSC and is applied to simulate strain path changes in low carbon steel. The path changes consist of tension followed by shear at different angles with respect to the preload direction, and forward simple shear followed by reverse shear. The results are compared to experimental data and highlight the role that directional dislocation structures induced during preload play during the reload stage.

\section{Introduction}

Recently, various microstructure-based models have been developed for simulating hardening response of metallic aggregates subjected to simple or complex strain paths. Yoon et al. (2005), Cheong et al. (2005), Vincze et al. (2005), Rauch et al. (2007), Beyerlein and Tomé (2007), Shiekhelsouk et al. (2009), Proust et al. (2009), Kysar et al. (2010), Lee et al. (2010), Rossiter et al. (2010), Hamelin et al. (2011), M’Guil et al. (2011), Rauch et al. (2011), Brown et al. (2012), and Segurado et al. (2012). The model proposed by Rauch et al. (2011), which was formulated for a continuous medium, follows the evolution of average dislocation populations divided in forward, latent and reversible. The motivation for the model is a key experimental observation, done in $\mathrm{Al}$ alloys and low carbon steels, of massive dislocation dissolution upon strain reloads, independently of temperature and of the character of the dislocation structure formed during preload.

The purpose of this paper is to reformulate the model proposed by Rauch et al. (2011) (referred to as RGBV from now on), within a crystallographic framework able to incorporate slip directionality at the grain level, texture effects, and an implicit way to account for strain path changes (as opposed to 'telling' the model when a path change occurs). The latter is an important feature, which should allow us to deal with multiple and arbitrary path changes.

The equations of the model are designed to capture the physical processes of dislocation accumulation/annihilation while remaining as simple as possible and converging towards the 'continuum' equations of RGBV for the case of monotonic loading. We avoid introducing detailed dislocation-dislocation interactions which are common in some crystallographic dislocation models (i.e. Franciosi et al., 1980; Tabourot et al., 1997; Lopes et al., 2003; Kubin et al., 2008; Devincre and Kubin,

\footnotetext{
* Corresponding author. Tel.: +351234370 827; fax: +351234370953.

E-mail address: jgracio@ua.pt (J.J. Gracio).
} 
2010; Bacroix and Brenner, 2012) because the corresponding hardening parameters and their evolution with strain become difficult to adjust independently. For this reason we keep the number of empirical parameters to a minimum while, at the same time, try to retain their connection with physical dislocation features. On the other hand, there are in the literature phenomenological attempts to mimic the material response under strain path changes (Barlat et al., 2011). The approach presented here represents a compromise between simplicity and retaining the crystallographic basis of plastic deformation.

\section{The RGBV model}

It is useful, before presenting our crystallographic model, to summarize the main concepts and equations associated with the RGBV. The model assumes an effective critical shear $\tau$ for activating dislocations, which hardens as a function of the accumulated shear $\Gamma$ in the grain, and is the same for every system of every grain. $\tau$ and $\Gamma$ are connected to the macroscopic flow stress and the strain increment through the average Taylor factor $M$, as:

$$
\begin{aligned}
& \Sigma=M \tau \\
& d \Gamma=M d \varepsilon
\end{aligned}
$$

The multiplicity and orientation of crystallographic systems is not accounted for, and texture is accounted for through the Taylor factor, but is not explicitly included in the model. The threshold stress is assumed to depend on the average dislocation density (the same in every grain) through the usual Taylor law

$$
\tau=\tau_{0}+\alpha \mu b \sqrt{\rho}
$$

The evolution of the dislocation density is given by the classic Kocks-Mecking law, with an athermal storage term and a temperature dependent recovery term

$$
d \rho=\frac{d \Gamma}{b \Lambda}-f \rho d \Gamma
$$

where $\Lambda$ is the dislocation mean free path. When a path change takes place, the reverse movement of most of the dislocations is temporarily facilitated leading to enhanced recovery. The model captures such effect by 'converting' a fraction $p$ of the dislocations present into 'reversible' dislocations:

$$
\begin{aligned}
& \rho_{\text {for }}=(1-p) \rho_{\text {for }, 0} \\
& \rho_{\text {rev }}=p \rho_{\text {for }, 0}
\end{aligned}
$$

where $\rho_{\text {for }, 0}$ is the dislocation density before reverse reload. The evolution of the dislocation densities is different for forward and reverse populations and is given by

$$
\begin{aligned}
& d \rho_{\text {for }}=\left(\frac{1}{b \Lambda}-f \rho_{\text {for }}\right) d \Gamma \\
& d \rho_{\text {rev }}=-\frac{1}{b \Lambda} \frac{\rho_{\text {rev }}}{\rho_{\text {for }, 0}} d \Gamma
\end{aligned}
$$

The forward dislocation population evolves as before, with storage and recovery, but the reversible dislocations can only decrease. The other extreme case of strain path change is 'orthogonal' reloading, and ideally corresponds to the case when, upon reload, the previously active systems become inactive, and previously non-active systems become active. In this case, the 'forward' dislocations become instantaneously 'latent' upon reload, as follows:

$$
\begin{aligned}
& \rho_{\text {for }}=0 \\
& \rho_{\text {lat }}=\rho_{\text {for }, 0}
\end{aligned}
$$

Upon reload, the forward dislocations evolve as before, and the latent dislocations only annihilate, as follows:

$$
\begin{aligned}
& d \rho_{\text {for }}=\left(\frac{1}{b \Lambda}-f \rho_{\text {for }}\right) d \Gamma \\
& d \rho_{\text {lat }}=-f \rho_{\text {lat }} d \Gamma
\end{aligned}
$$

When the reload is neither exact reversal or ideally orthotropic, RGBV's model uses a conversion between forward, reversible and latent dislocations based on a 'path change index' initially proposed by Schmitt et al. (1994). The 'path change index' is defined as the projection of the reload strain tensor upon the preload tensor, and varies from 1 (no path change) to - 1 (path reversal), passing through 0 (orthogonal path change). In the most general case the threshold stress depends on all the dislocation populations as

$$
\tau=\tau_{0}+\alpha \mu b \sqrt{\rho_{\text {for }}+\rho_{\text {rev }}+L \rho_{\text {lat }}}
$$

where $L$ is a latent hardening parameter, usually larger than one. Observe that the decision about converting dislocation populations is made using a criterion based on changes in the macroscopically imposed strain rate. 


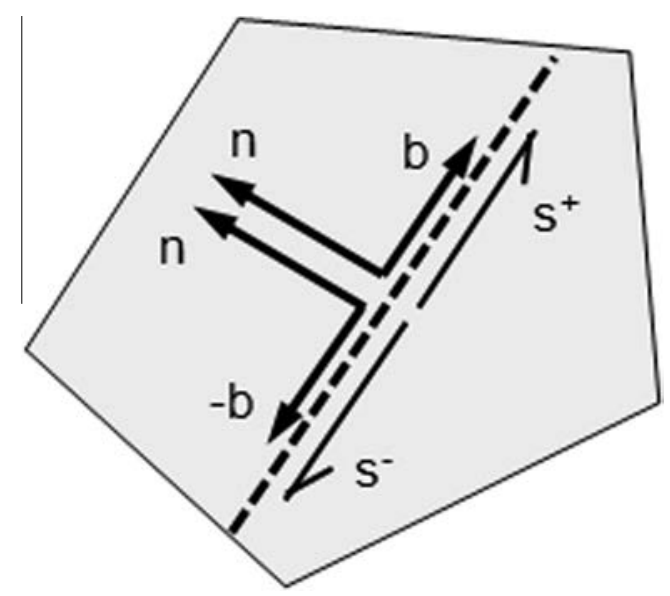

Fig. 1. Schematic illustration defining direct and opposite systems $s^{+}$and $s^{-}$.

\section{Crystallographically based dislocation model}

The crystallographic version of the RGBV model presented here relies on the same conceptual idea that strain path changes progressively 'erase' the previously stored dislocations, independently of the specific structures that were formed previously. The crystallographic model defines similar parameters as the continuous model, but differs from the latter in that it follows forward and reversible dislocations in the individual slip systems of the grain. While the math is slightly more involved, the advantage is that texture and directionality effects are accounted for by accounting for grain orientation, and reversible or latent dislocations are explicitly related to the shear activity in each slip system.

In order to consider reversible dislocation densities, we associate with each slip system two systems $s^{+}$and $s^{-}$that have the same slip plane normal $\mathbf{n}$, but opposite (arbitrarily defined) slip directions $\mathbf{b}$ and $-\mathbf{b}$ (see Fig. 1). In our model these conjugate systems cannot be simultaneously active, and the assignment of $s^{+}$and $s^{-}$is arbitrary, since the sense of shear will vary depending on the deformation mode imposed to the aggregate. We define the dislocation density on a system $s$ as the sum of a forward ${ }^{1}$ density, common to $s^{+}$and $s^{-}$, and a reversible density specific to $s^{+}$and $s^{-}$:

$$
\rho^{s}=\rho_{\mathrm{for}}^{s}+\rho_{\text {rev }}^{s^{+}}+\rho_{\text {rev }}^{s^{-}}
$$

The total dislocation density in a grain is given by the sum of forward and reversible on all systems

$$
\begin{aligned}
& \rho=\sum_{s} \rho^{s} \\
& \rho=\rho_{\text {for }}+\rho_{\text {rev }}=\sum_{s} \rho_{\text {for }}^{s}+\sum_{s}\left(\rho_{\text {rev }}^{s^{+}}+\rho_{\text {rev }}^{s^{-}}\right)
\end{aligned}
$$

The CRSS of slip system $s$ is related to dislocation density through the usual Taylor law

$$
\tau^{s}=\tau_{0}+\alpha \mu b \sqrt{\rho^{s}+L \sum_{s^{\prime} \neq s} \rho^{s^{\prime}}}
$$

where $L$ is a latent hardening parameter. We would like to point out that Eq. (11) assumes that any dislocation $s^{\prime}$ that is not on system $s$ will interact with $s$ independently of their relative configurations. This is clearly not correct for non-cubic materials, and even for cubic structures this is a rough approximation. Some authors (Peeters et al., 2000, 2001a,b; Kubin et al., 2008) have explored more complex interactions of the form

$$
\tau^{s}=\tau_{0}+\mu b \sqrt{\alpha^{s s} \rho^{s}+\sum_{s^{\prime} \neq s} \alpha^{s^{\prime}} \rho^{s^{\prime}}}
$$

While certainly more accurate, Eq. (12) adds 6 parameters for FCC (Madec and Kubin, 2008), 5 parameters for BCC (Queyreau et al., 2009) and 15 parameters for HCP (Capolungo, 2011). Such number of parameters and their possible evolution with strain are difficult to assess independently and conspire against the simplicity of the model. In addition, Eq. (11) assigns the same threshold stress for activation of $s^{+}$and $s^{-}$dislocations. This amounts to disregarding internal back stresses in the grain which induce an apparent lowering of the CRSS when the sense of shear is reversed, and lead to a

\footnotetext{
1 While the expression non-reversible dislocations would also be appropriate, in what follows we will refer to those dislocations 'locked' inside the grain as 'forward' dislocations, and use the subscript 'for' to designate them (not to be confused with 'forest' dislocations, which is a different concept). The dislocations called 'reversible' are those that can glide (and recombine) in the opposite direction following an inversion of the resolved shear that generated them.
} 
Bauschinger effect upon unloading. The Bauschinger effect takes place in a small strain interval (about 1-2\%), is driven by internal stresses, and has associated a large elasto-plastic hardening rate. Our model does not attempt to capture the elasto-plastic portion of the reload curve. Instead, it focuses on the reload response characterized by a large strain plateau with a low or even zero hardening rate.

In what follows we define the mean free path for dislocations as

$$
\frac{1}{\Lambda}=\frac{\sqrt{\rho}}{K}+\frac{1}{D}
$$

$D$ is the grain size and $K$ is the average number of immobile dislocations that a mobile dislocation will cut through before itself becoming immobile. Observe that we use the total dislocation density in Eq. (13) because mobile dislocations will cut both, forward and reversible non-coplanar dislocations. We define the total shear increment in the grain as the sum over the shears contributed by every active system

$$
d \Gamma=\sum_{s}\left|\mathrm{~d} \gamma^{s}\right|
$$

For forward dislocation densities, the evolution law is given by the classic Kocks-Mecking expression containing a creation and an annihilation term. For reverse dislocation densities, the evolution law should account for the creation of potentially reversible dislocations in the active system and for the annihilation of reversible dislocations in the opposite system, provided that the latter are present. Three different situations are possible for the evolution of dislocations associated with system $s$ :

$$
\text { Case1 : } \begin{aligned}
d \gamma^{s^{+}} & >0\left(\Rightarrow d \gamma^{s^{-}}=0\right) \\
d \rho_{\mathrm{for}}^{s} & =(1-p) \frac{d \gamma^{s^{+}}}{b \Lambda}-f \rho_{\mathrm{for}}^{s} d \Gamma \\
d \rho_{\mathrm{rev}}^{s^{+}} & =p \frac{d \gamma^{s^{+}}}{b \Lambda}-f \rho_{\mathrm{rev}}^{s^{+}} d \Gamma \\
d \rho_{\mathrm{rev}}^{s^{-}} & =-\frac{1}{b \Lambda} \frac{\rho_{\mathrm{rev}}^{s^{-}}}{\rho_{0}^{s}} d \gamma^{s^{+}}
\end{aligned}
$$

We use $d \Gamma$ instead of $d \gamma^{s^{+}}$in the recombination term, because activity in systems other than $s$ will induce 'indirect recombination' in $s$ by an amount proportional to the density of $s$-dislocations, and to the shear taking place on those other systems (Rauch et al., 2011). Observe that Eq. (15) partitions the dislocations created into 'forward' and 'reversible', through the proportionality factor ' $p$ '. In the case of monotonic loading, forward and reversible add up to the total dislocation density and are indistinguishable as far as hardening is concerned. During 'forward' loading $d \gamma^{s^{+}}>0$ we allow for the simultaneous creation in the grain of potentially reversible dislocations $\rho_{\text {rev }}^{s^{+}}$(which will be 'used' during 'reverse' loading $d \gamma^{s^{-}}>0$ ) and for the recombination of reversible dislocations $\rho_{\text {rev }}^{s^{-}}$(which may be left from previous activity $d \gamma^{s^{-}}>0$ ). It seems logical that the rate of recombination of reversible dislocations is assumed to be proportional to the shear $d \gamma^{s^{+}}$. The 'utilization factor' $0 \leqslant \rho_{\text {rev }}^{s^{-}} / \rho_{0}^{s} \leqslant 1$ is proportional to the updated reversible density $\rho_{\text {rev }}^{s^{-}}$and inversely proportional to the total density $\rho_{0}^{s}$ that was present when the shear was reversed in system ' $s$ '. The effect of this term is to reduce the total dislocation density in the grain and so to reduce the threshold stress (Eq. (12)) for the system. The physical justification for this normalization is given in RGBV: the 'unstorage' rate promoted by dislocations moving backward is bounded by the storage rate they produced when they were moving forward (Rauch et al., 2007). For example, a gliding segment leaves loops surrounding the obstacles located on the slip plane. When glide is reversed, the same segment will fully or partially destroy the same loops. Consequently, the reverse annihilation process scales with the forward storage. RGBV use the forward density previous to reloading in the denominator, and we use the total density previous to shear inversion. Since $p=1$ up to moderate strains, it turns out that both expressions are equivalent: for us the total equals the reversible if $p=1$, and RGBV convert all the forward population into reversible if $p=1$.

$$
\text { Case2 : } \begin{aligned}
d \gamma^{s^{-}} & >0\left(\Rightarrow d \gamma^{s^{+}}=0\right) \\
d \rho_{\mathrm{for}}^{s} & =(1-p) \frac{d \gamma^{s^{-}}}{b \Lambda}-f \rho_{\mathrm{for}}^{s} d \Gamma \\
d \rho_{\mathrm{rev}}^{s^{+}} & =-\frac{1}{b \Lambda} \frac{\rho_{\mathrm{rev}}^{s^{+}}}{\rho_{0}^{s}} d \gamma^{s^{-}} \\
d \rho_{\mathrm{rev}}^{s^{-}} & =p \frac{d \gamma^{s^{-}}}{b \Lambda}-f \rho_{\text {rev }}^{s^{-}} d \Gamma
\end{aligned}
$$

This case, which is the equivalent of Case 1 when shear takes place on $s^{-}$, is listed here separately in order to reflect the fact that numerically one needs to keep track separately of $d \gamma^{s^{+}}>0$ and $d \gamma^{s^{-}}>0$. We generate reversible dislocations on $s^{-}$ and simultaneously use available reversible dislocations on $s^{+}$. 


$$
\text { Case3 : } \begin{aligned}
d \gamma^{s^{+}} & =0, d \gamma^{s^{-}}=0 \\
d \rho_{\mathrm{for}}^{s} & =-f \rho_{\mathrm{for}}^{s} d \Gamma \\
d \rho_{\text {rev }}^{s^{+}} & =-f \rho_{\text {rev }}^{s^{+}} d \Gamma \\
d \rho_{\text {rev }}^{s^{-}} & =-f \rho_{\text {rev }}^{s^{-}} d \Gamma
\end{aligned}
$$

In this case systems $s^{+}$or $s^{-}$are not active, but 'indirect' annihilation due to activity in other systems will reduce both, the forward and the reversible densities on them. This is a consequence of making annihilation proportional to the total slip activity $d \Gamma$. Another consequence of this assumption concerns the recombination factor ' $f$. Observe that we do not propose a different factor $f_{\text {latent }}$ in Eq. (17) because in Eqs. (15) and (16) ' $f$ ' already stands for all the annihilation events, not only 'selfannihilation'.

\subsection{The monotonic loading case}

Here we apply the equations above to the case of monotonic load. When load is initially applied we can arbitrarily label all active shears in the grain as $s^{+}$, such that all $d \gamma^{s^{+}}>0$ and $d \gamma^{s^{-}}=0$. In addition, because initially $\rho_{\text {rev }}^{s^{+}}=\rho_{\text {rev }}^{s^{-}}=0$, the evolution laws lead to a monotonic build-up of dislocations $\rho_{\text {rev }}^{s^{+}} \geqslant 0$ and $\rho_{\text {rev }}^{s^{-}}=0$. These conditions are represented by Cases 1 and 3. Adding Eqs. (15) and (17) for all systems we get the total increment for the grain:

$$
\begin{aligned}
& d \rho=\sum_{s} d \rho^{s}=\sum_{s}\left(d \rho_{\mathrm{for}}^{s}+d \rho_{\text {rev }}^{s^{+}}\right) \\
& d \rho=\frac{(1-p)}{b \Lambda} \sum_{s \text { active }} d \gamma^{s^{+}}-f d \Gamma \sum_{s \text { active }} \rho_{\text {for }}^{s}+\frac{p}{b \Lambda} \sum_{s \text { active }} d \gamma^{s^{+}}-f d \Gamma \sum_{s \text { active }} \rho_{\text {rev }}^{s^{+}}-f d \Gamma \sum_{s \text { inactive }} \rho_{\text {for }}^{s}-f d \Gamma \sum_{s \text { inactive }} \rho_{\text {rev }}^{s^{+}} \\
& \left.\left.d \rho=\frac{1}{b \Lambda} \sum_{s \text { active }} d \gamma^{s^{+}}-f \sum_{s \text { active }} \rho_{\text {for }}^{s}+\sum_{s \text { inactive }} \rho_{\text {for }}^{s}\right) d \Gamma-f \sum_{s \text { active }} \rho_{\text {rev }}^{s^{+}}+\sum_{s \text { inactive }} \rho_{\text {rev }}^{s^{+}}\right) d \Gamma
\end{aligned}
$$

In this limit case (we assume no previously stored dislocations in the currently inactive slip systems) we recover the simpler evolution equation of the continuum model (RGBV, Eq. (13)) proposed for the representative medium

$$
d \rho=\frac{1}{b \Lambda} d \Gamma-f\left(\rho_{\text {for }}+\rho_{\text {rev }}\right) d \Gamma
$$

This conclusion is reassuring, since such simple form could already explain several features associated with reloading. What our new model adds is the directionality associated with tracking dislocations densities in individual planes and with the crystallographic orientation of the crystal with respect to the loading axis. In addition, reversibility and latent hardening are implicitly included in the model through the activity of the slip systems.

\subsection{Recombination parameter $f$}

As discussed above, we use a unique recombination parameter ' $f$ '. This of course is a simplification, since one would expect this parameter to depend on the relative orientation and character of the dislocation segments that recombine. For example, one may expect recombination by cross slip of screw components of the same system to be more efficient than recombination between non-colinear slip systems. A systematic characterization of these processes would require a basic dislocation interaction analysis and would increase substantially the complexity of the model. We expect this parameter to play a more critical role in hexagonal than in cubic materials, because the reactions between the different deformation modes (such as prism, basal, pyramidal) can be expected to be very different. The reader can consult papers by Kubin et al. (2008), Madec and Kubin (2008), Queyreau et al. (2009), Balint et al. (2008), Weinberger (2011), Capolungo (2011) for Molecular Dynamics and Discrete Dislocation Dynamics estimates of coupling parameters in cubic and hexagonal crystals.

\subsection{Reversibility parameter $p$}

The reversibility parameter ' $p$ ' represents the fraction of the stored dislocations which are loosely tangled and can be activated in the opposite sense upon shear stress reversal on the system. While the continuous model of RGBV assigns a constant value to the reversibility parameter ' $p$ ', it is evident that as strain accumulates more obstacles (debris) are left in the grain which will hinder the capability of dislocations to glide in the opposite sense. As a consequence, $p$ should evolve monotonically with strain from $p=1$ towards $p=0$. The former case applies to small strains, when all stored dislocations can be reversed, and the latter case corresponds to large strain, when debris prevents dislocations to annihilate when gliding in the opposite sense.

It is possible to estimate this fraction and its evolution with strain using shear reversal tests. Basically, the value of $p$ is related to the strain shift required to superimpose the monotonic stress-strain curve with the one associated with shear 

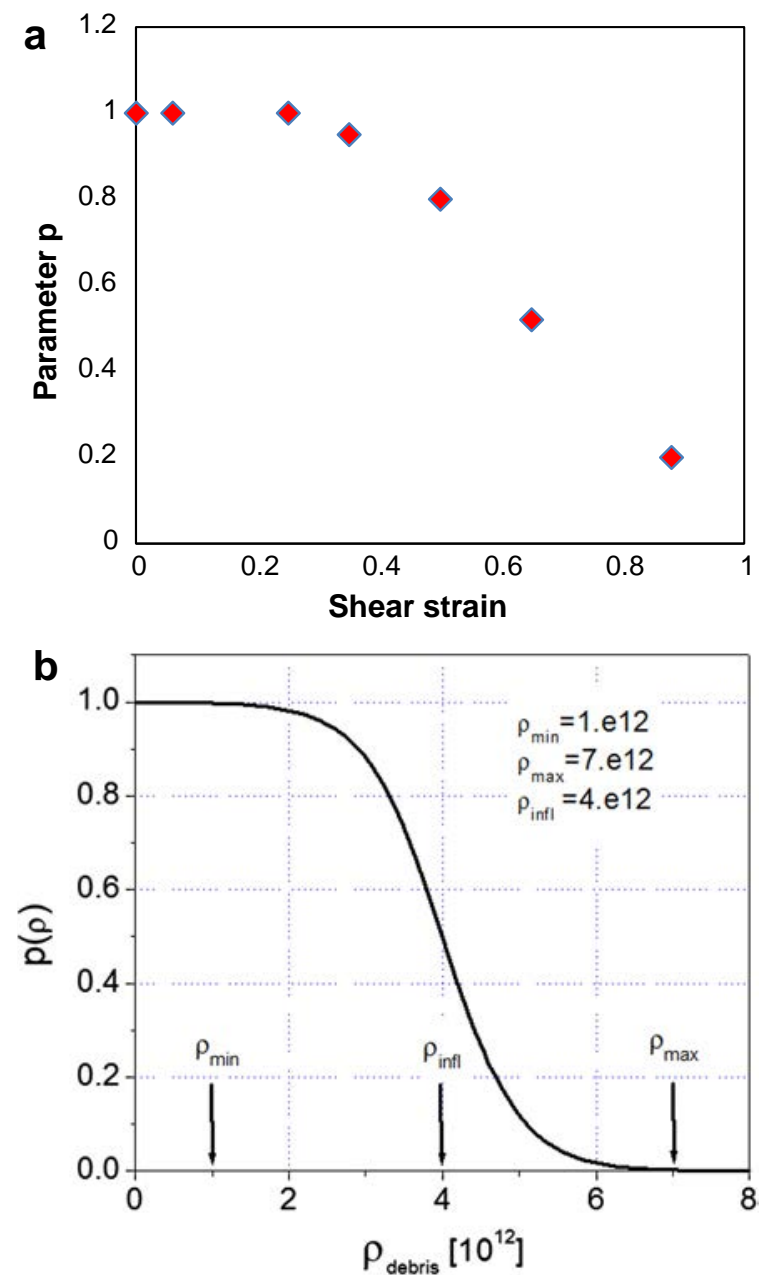

Fig. 2. (a) Empirical estimate of the reversibility parameter for the case of shear/reverse shear (see Appendix A for details); (b) dependence of the reversibility parameter $p$ with debris density.

reloads in the opposite direction. The procedure is detailed in Appendix A where the shear results related to low carbon steel are used. Such result suggests that reversible dislocations are still formed up to shear strains close to one (see Fig. 2a).

Here we argue that the movement of dislocations is impeded by debris. Since debris is produced by incomplete annihilation, then the rate of debris accumulation should scale with the dislocation recovery term $(f \rho d \Gamma)$ in the evolution equations (Eqs. (15)-(17)). A loose estimate of debris density would be given by:

$$
\rho_{\text {deb }}=\int_{0}^{\varepsilon} \rho d \Gamma=\int_{0}^{\varepsilon}\left(\sum_{s} \rho^{s}\right) d \Gamma
$$

This estimate is similar to the one proposed by Rollett et al. (1987) for explaining Stage IV work hardening. In addition, we know that the recovery factor should decrease monotonically as debris increases, in the fashion shown in Fig. 2a. As a consequence, in this paper we propose an empirical sigmoid dependence of the recovery parameter with $\rho_{\text {deb }}$, of the form

$$
P_{\left(\rho_{\text {deb }}\right)}=0.5-0.5 \tan h\left[3\left(\frac{\rho_{\max }+\rho_{\min }}{\rho_{\max }-\rho_{\min }}\right)\left(\frac{\rho_{\text {deb }}-\rho_{\text {infl }}}{\rho_{\text {infl }}}\right)\right]
$$

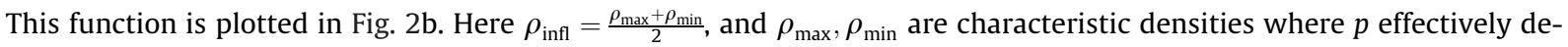
parts from 1 and where it becomes effectively zero, respectively. They may be regarded as material properties, although here we assign values based on experiment fits to the empirical estimate of Fig. 2a. The value of $p$ controls how rapidly each grain goes from generating mostly reversible dislocations to shutting down the production of reversible dislocations.

Eq. (20) tells us that, even if the dislocation density saturates (as is the case with the Kocks-Mecking law), $\rho_{\text {deb }}$ will keep increasing with deformation. The empirical law equation. (21), on the other hand, guarantees that in such a case $p$ will go asymptotically to zero anyway. Notice that this is the only use of the debris density in the model, and we do not explicitly 
account for it in the evolution equations. If one were to do so one would obtain a Stage IV hardening at large strains associated with the non-zero debris production. A model accounting for the effect of debris (as defined by Eq. (20)) on hardening, but without reversible dislocations, was proposed recently by Beyerlein and Tomé (2008) for hexagonal materials.

Another characteristic of the model presented here is that the factor $p$ will evolve differently from grain to grain, depending on the history of slip activity in the grain. For such reason, in what follows we present results for the grain average of $p$, and for the standard deviation of $p$ within the grain population forming the aggregate. If we call $p^{g}$ the value of $p$ in grain $g$, and if $\omega^{g}$ is the volume fraction associated with the grain, the standard deviation is given by

$$
\sigma_{P}=\sqrt{\sum_{g}\left(p^{g}-\bar{p}\right)^{2} \omega^{g}}
$$

\section{Visco-plastic self-consistent polycrystal model}

The single crystal formulation discussed in Section 3 relates the threshold stress on the slip systems of the crystal to the associated dislocation densities (Eq. (11)), and relates the evolution of the dislocation densities to the shear activity of the different systems (Eqs. (15)-(17)). In this section we relate the deformation (due to crystallographic shear) of the individual grain to the macroscopic strain imposed to the polycrystal. Here, we implement the single crystal hardening law into the visco-plastic self-consistent (VPSC) polycrystal model originally proposed by Molinari et al. (1987) and later extended to fully anisotropic behavior and arbitrary crystal symmetry by Lebensohn and Tomé (1993). Only a brief qualitative description of the model is given below. A comprehensive description can be found in Lebensohn et al. (2007).

\subsection{Brief description of VPSC}

A polycrystal model represents the material as a collection of crystal orientations with assigned weights: the orientations represent grains and the weights represent the associated volume fractions. The latter are chosen to reproduce the initial texture of the material. Within the VPSC model each grain is treated as an ellipsoidal visco-plastic inclusion embedded in an effective visco-plastic medium which represents the 'average' environment 'seen' by each grain. Both, inclusion and medium, have fully anisotropic properties. The grain deforms by shear on slip systems activated by a Resolved Shear Stress, and the deformation of the grain follows from its interaction with the effective medium. Linearization of the constitutive response guarantees us that stress and strain rate are uniform within the ellipsoidal domain, although they are different for different grains, depending on the relative anisotropy between grain and medium.

The strain rate of the grain is given by the sum of shear rates $\dot{\gamma}^{s}$ over the active systems. The latter are described by a visco-plastic non-linear constitutive equation:

$$
\dot{\varepsilon}_{i j}^{g}=\sum_{s} m_{i j}^{s} \dot{\gamma}^{s}=\dot{\gamma}_{o} \sum_{s} m_{i j}^{s}\left(\frac{m_{k l}^{s} \sigma_{k l}^{g}}{\tau^{s}}\right)^{n}
$$

In the above expression $\tau^{s}$ is the threshold stress required to activate system $s$, and its evolution with strain is the focus of this paper; $m_{i j}^{s}=\frac{1}{2}\left(n_{i}^{s} b_{j}^{s}+n_{j}^{s} b_{i}^{s}\right)$ is the symmetric Schmid tensor associated with slip system $s ; \bar{n}^{s}$ and $\bar{b}^{s}$ are the normal and Burgers vector of the system, $\dot{\varepsilon}_{i j}^{g}$ and $\sigma_{k l}^{g}$ are the deviatoric strain-rate and stress of the grain; $\dot{\gamma}_{o}$ is a normalization rate and $n$ is the rate-sensitivity exponent, taken here as $1 / \mathrm{s}$ and 20 , respectively. The linearized form of the single crystal response is:

$$
\dot{\varepsilon}_{i j}^{g}=M_{i j k l}^{g}\left(\sigma^{g}\right) \sigma_{k l}^{g}+\dot{\varepsilon}_{i j}^{0, g}
$$

where $M_{i j k l}^{g}$ and $\dot{\varepsilon}_{i j}^{o . g}$ are the viscoplastic compliance and the back-extrapolated term of grain $g$, respectively. The linearization chosen for our simulation is $n^{\text {eff }}=10$, which gives a response in between the stiff secant and soft tangent approximations (Lebensohn et al., 2007). Performing homogenization on this linearized heterogeneous medium consists in assuming a linear relation analogous to Eq. (24) at the effective medium (polycrystal) level:

$$
\dot{E}_{i j}=\bar{M}_{i j k l} \Sigma_{k l}+\dot{E}_{i j}^{o}
$$

where $\dot{E}_{i j}$ and $\Sigma_{i j}$ are the macroscopic rate and stress, and $\bar{M}_{i j k l}$ and $\dot{E}_{i j}^{o}$ are the macroscopic viscoplastic compliance and back extrapolated term, respectively. The latter moduli are unknown a priori and need to be adjusted self-consistently.

\subsection{Simulation conditions}

In the simulations, deformation is imposed incrementally by enforcing a macroscopic velocity gradient $L_{i j}=\partial \dot{U}_{i} / \partial X_{j}$ during a time interval $\Delta t$. For axial tension we enforce $L_{11}=10^{-3} \mathrm{~s}^{-1}, \Delta t=1 \mathrm{~s}$, and stress-free conditions $\Sigma_{22}=\Sigma_{33}=0$ on the lateral surfaces of the sample. For shear test simulations we enforce $L_{12}=10^{-3} \mathrm{~s}^{-1}$, the remaining $L_{i j}=0$, and $\Delta t=1 \mathrm{~s}$.

In the case of the rolled and recrystallized low carbon steel analyzed here we use 1000 grains to represent the aggregate. The initial texture associated with this collection of grains is depicted in Fig. 3 as pole figures. 


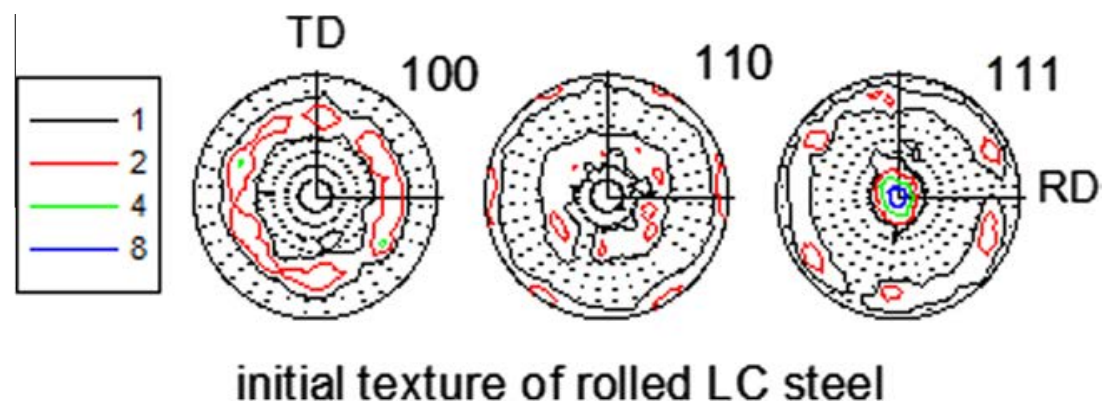

Fig. 3. Pole figures associated with the 1000 grains used in this work as starting texture for LC.

At the crystal level we assume that deformation is accommodated by slip on the $\{110\}\langle 111\rangle$ and $\{112\}\langle 111\rangle$ slip modes characteristic of BCC structures. Each mode comprises 12 slip systems and the corresponding hardening parameters, adjusted to the experimental data, are listed in Table 1.

Although these parameters may be different for each mode, here only the initial threshold stress $\tau_{0}$ in each system (Eq. (11)) is allowed to vary from mode to mode. The reason is two-fold: we want to keep the model as simple as possible, and we do not have sufficient microstructural information for linking the parameters to specific dislocation reactions and interactions.

We use two sets of available strain-path-change experiments for benchmarking our model: the first set consists of three shear experiments done on samples cut from rolled LC steel, to preloads of $\varepsilon_{12}=\gamma_{12} / 2=10 \%, 20 \%$, 30\%, followed by shear in the opposite sense (axis 1 is the RD and axis 2 is the TD of the sheet). The stress-strain response is shown in Fig. 4 (Rauch et al., 2007).

Table 1

Parameters of the hardening model for LC steel \#1 and LC steel \#2.

\begin{tabular}{lll}
\hline Dislocation model & Steel \#1 (shear/shear) & Steel \#2 (tension/shear) \\
\hline$\mu$ (Elastic shear modulus) & $85 \mathrm{GPa}$ & $85 \mathrm{GPa}$ \\
$B$ (Burgers vector) & $2.4610^{-10} \mathrm{~m}$ & $2.4610^{-10} \mathrm{~m}$ \\
$D$ (Grain size) & $3010^{-6} \mathrm{~m}$ & $3010^{-6} \mathrm{~m}$ \\
$\alpha$ (Dislocation-dislocation interaction) & 0.50 & 0.50 \\
$\tau_{0}$ (Initial CRSS for $\left.\{110\}\langle 111\rangle\right)$ & $55 \mathrm{MPa}$ & $38 \mathrm{MPa}$ \\
$\tau_{0}$ (Initial CRSS for $\left.\{112\}\langle 111\rangle\right)$ & $55 \mathrm{MPa}$ & $40 \mathrm{MPa}$ \\
$K$ (Mobile-to-storage parameter) & 320 & 950 \\
$f$ (Recombination parameter) & 1.7 & 1.7 \\
$L$ (Latent hardening parameter) & 1.6 & 2.1 \\
$\rho_{\min }$ (Lower reversibility threshold) & $410^{12} \mathrm{~m}^{-2}$ & $410^{12} \mathrm{~m}^{-2}$ \\
$\rho_{\max }$ (Upper reversibility threshold) & $26010^{12} \mathrm{~m}^{-2}$ & $26010^{12} \mathrm{~m}^{-2}$ \\
\hline
\end{tabular}

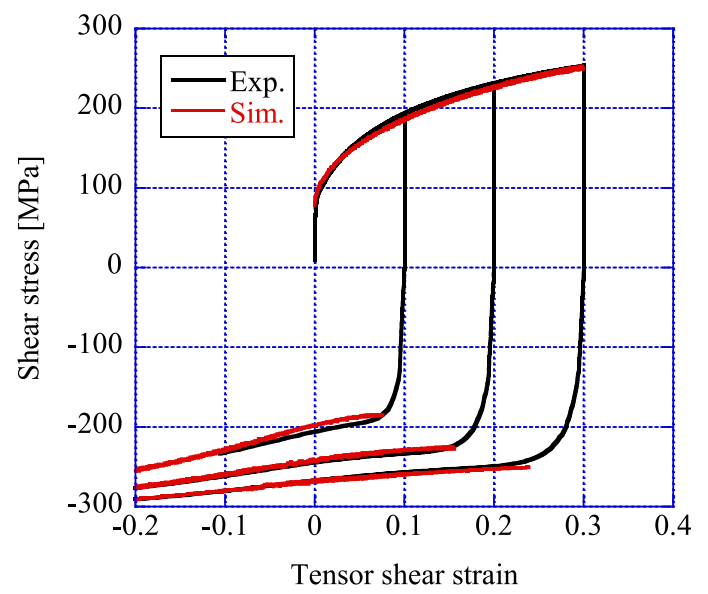

Fig. 4. Stress-strain response associated with $10 \%, 20 \%$ and $30 \%$ shear preload in the Rolling Direction followed by reverse shear. 
The second set consists of three tensile experiments done on flat dog-bone specimens with a large gauge section, to preloads of $\varepsilon_{11}=5 \%, 10 \%, 20 \%$ (axis 1 is the RD of the sheet). Shear samples were cut from each of the preloaded gauge regions at $45^{\circ}, 90^{\circ}, 135^{\circ}$ and a shear strain $\varepsilon_{12}=\gamma_{12} / 2$ was imposed along the longitudinal axis of the samples. The stress-strain response is shown in Fig. 5 (Rauch and Schmitt, 1989). It is unfortunate that each set of experiments was performed on LC steels of different pedigree, which means that some of the hardening parameters differ between set \#1 and set \#2 (see Table 1).

The first set of experiments, shear/reverse shear, is the most straightforward case of strain reversal. In what concerns the second set, a state of tension along 1 with contraction along 2 and 3 represents shear when rotated by $45^{\circ}$ around axis 2 or 3 . The $135^{\circ}$ shear reload amounts to a reversal in the 1-2 plane (but not in the 1-3 plane), the $90^{\circ}$ case represents 'orthogonal
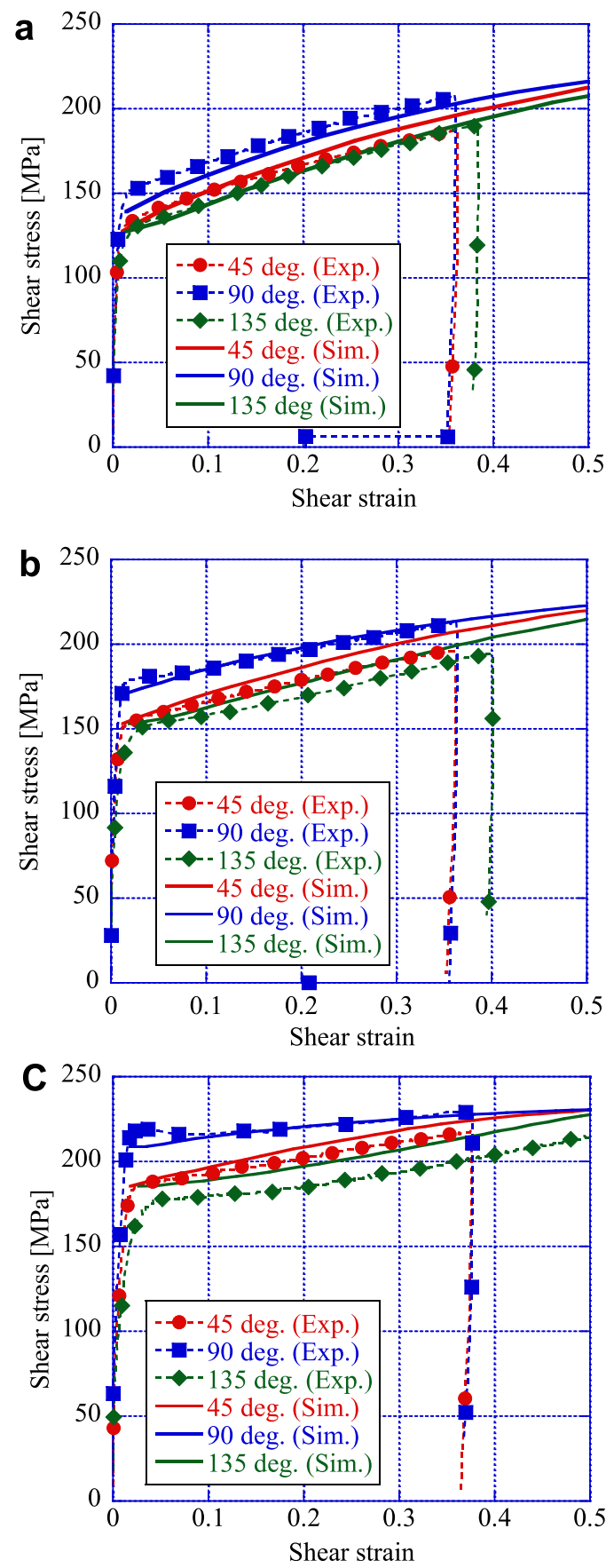

Fig. 5. Shear stress vs. shear strain, following preloading in tension along the RD by (a) $5 \%$; (b) $10 \%$; (c) $20 \%$. In each case shear reloads were imposed at $45^{\circ}$, $90^{\circ}$ and $135^{\circ}$ with respect to the preload direction. 
loading, where active systems during pre-load become non-active (and vice versa), and the $45^{\circ}$ case represents 'quasimonotonic' shear in the 1-2 plane. As can be seen from the experiments the $135^{\circ}$ reloads exhibit the most softening, and the orthogonal reloads the most hardening, as one would expect.

\section{Results and discussion}

In addition to the stress-strain response of the aggregate, a polycrystal simulation provides information about stress, slip activity and dislocation density in individual grains. Polycrystal models also provide information about texture and overall dislocation density evolution, which can be compared to experimental measurements. In our simulations we follow the dislocation density associated with each system in each grain, but here we only report averages of 'forward' and 'reversible' dislocations. We also report the relative contribution to deformation of slip modes $\{110\}$ and $\{112\}$, averaged over all grains.

The visco plastic model used here does not account for elastic effects or internal back-stresses. As a consequence, the simulations do not capture the elasto-plastic transition and the Bauschinger effect that takes place during the initial few
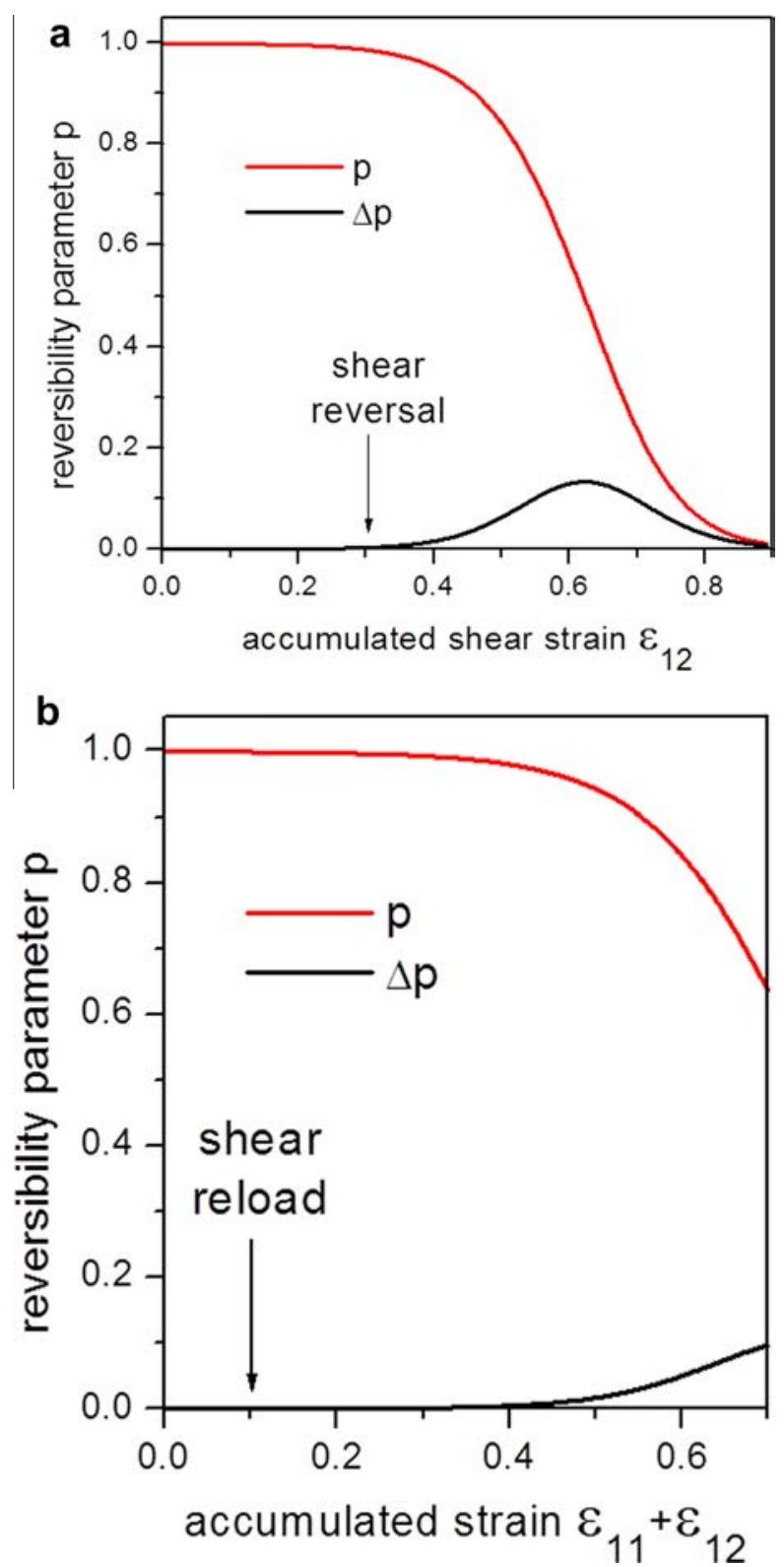

Fig. 6. Evolution of the reversibility ' $p$ ' and its standard deviation with strain for the case of (a) $30 \%$ shear preload followed by reverse shear; (b) $10 \%$ tension preload along RD followed by shear reload at $90^{\circ}$. 
percents of reload strain. Therefore, in order to compare predicted and measured stress-strain curves, we arbitrarily define the end of the elasto-plastic transition as the strain at which the experimental flow curve has a slope of $1 \mathrm{GPa}$, and shift the predicted flow curves by such amount (see Figs. 4 and 5).

\subsection{Shear preload followed by reverse shear}

Fig. 4 shows the measured and predicted stress response for the three shear reversals. The simulation captures very well the extended plateau in the reload curves. According to our calculations, such response can be explained only if the totality of dislocations generated during preload can be reversed (i.e., $p=1$ ), even after 30\% preload strain. Figs. 6 and 7 a illustrate the case: only after $30 \%$ the reversibility parameter starts to decrease and 'forward' dislocations start being generated. Two things happen following load reversal $\left(d \gamma^{s^{-}}>0\right)$ : previously generated reversible dislocations $\rho_{\text {rev }}^{s^{+}}$recombine while forward dislocations $\rho_{\text {for }}^{s}$ and reversible dislocations $\rho_{\text {rev }}^{s-}$ are simultaneously being generated. The balance is such that it prevents the flow stress from increasing for about 20\% reload strain (see Fig. 4). Eventually, the $\rho_{\text {rev }}^{s^{+}}$are being exhausted and the flow stress increases. However, the $\rho_{\text {rev }}^{s^{-}}$would be able to recombine and keep the flow stress from increasing if a second shear reversal would be applied to the sample. The decrease with strain of the $p$ parameter, will favor the production of forward dislocations and diminish the formation of reversible dislocations, with the consequence that stress-strain plateaus will tend to disappear during shear cycling. Observe that the standard deviation in the $p$ parameter is roughly $13 \%$ at its maximum, indicating that, despite the nearly 'plastically isotropic' character of the BCC lattice, the population of reversible dislocations varies with grain orientation.

Fig. 8a depicts the relative contribution of the two slip modes, and shows how the presence of an initial texture biases the slip activity. Observe that both, forward and reverse shear, are mostly accommodated by $\left\{\begin{array}{llll}1 & 1 & 0\end{array}\right\}\left\langle\begin{array}{lll}1 & 1 & 1\end{array}\right\rangle$ slip $(\sim 70 \%)$, despite the fact that $\{112\}\langle 111\rangle$ slip ( $30 \%)$ also comprises 12 slip systems. It seems apparent, then, that the response associated with this case is mostly controlled by $\{110\}$ systems and their associated hardening parameters.
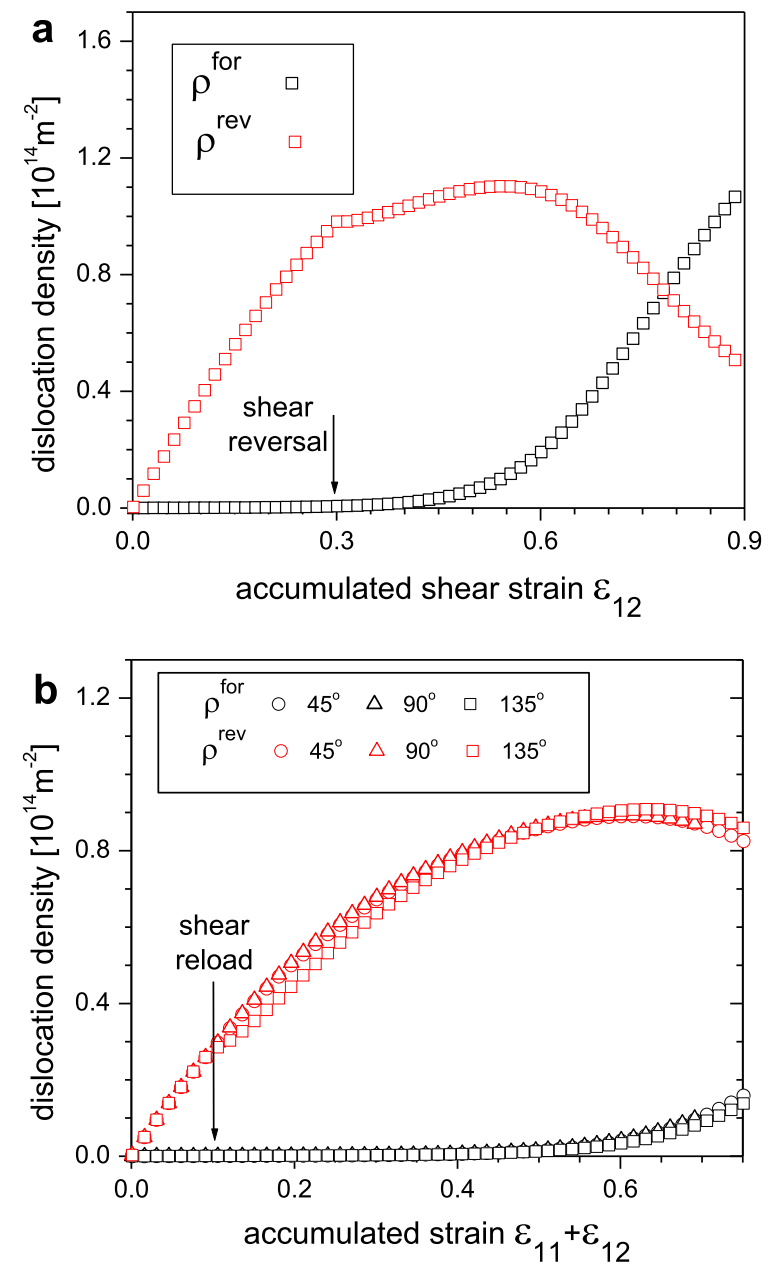

Fig. 7. Evolution of forward and reverse dislocation density during (a) $30 \%$ shear preload followed by reverse shear; (b) $10 \%$ tensile preload along RD followed by shear reload at $90^{\circ}$. 

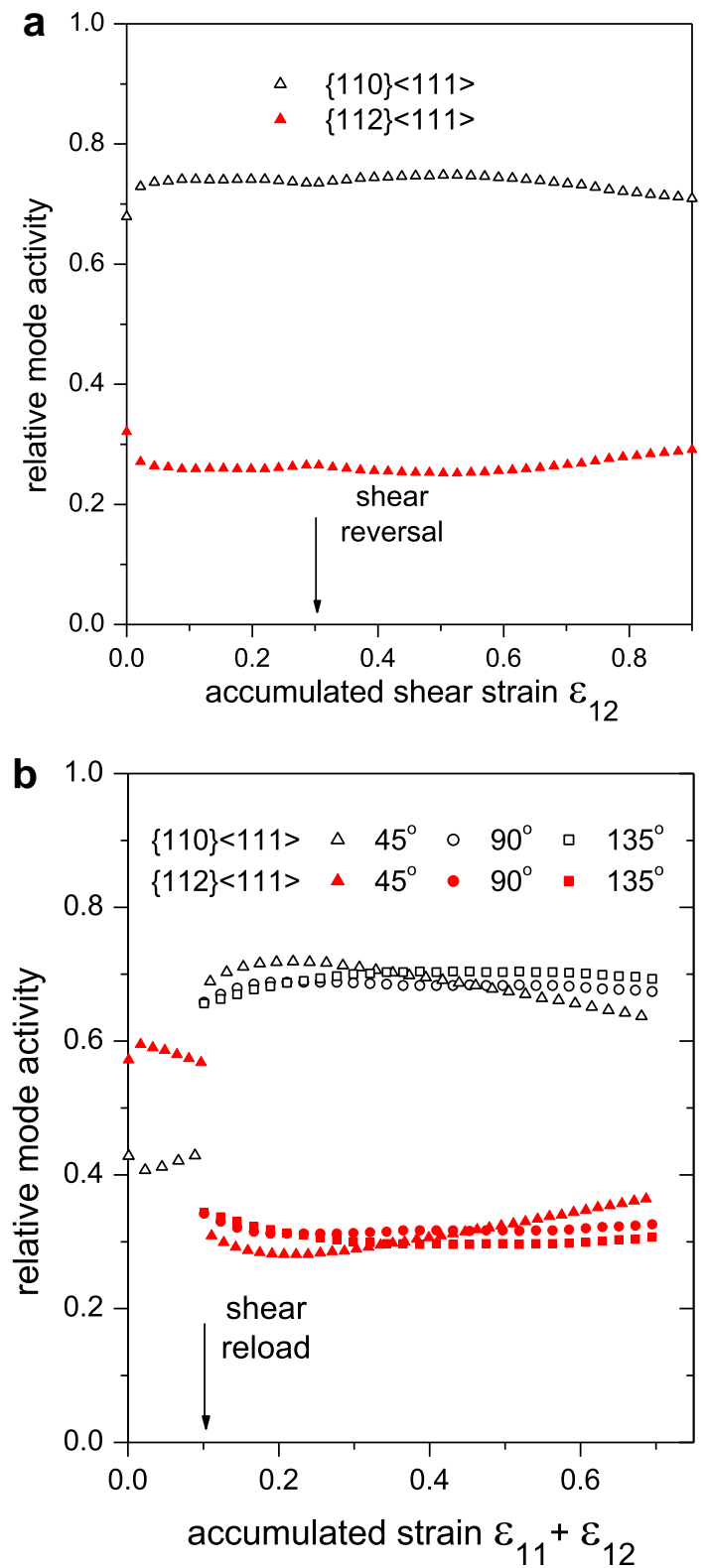

Fig. 8. Relative contribution of $\{110\}$ and $\{112\}$ slip to deformation during (a) $30 \%$ shear preload followed by reverse shear; (b) $10 \%$ tension preload followed by shear at $45^{\circ}, 90^{\circ}, 135^{\circ}$.

Concerning texture, Fig. 9a-c shows the formation of characteristic shear components during the $30 \%$ shear preload, a reversal towards the initial rolling texture after $30 \%$ reverse shear reload, and the formation of shear components after extra $30 \%$ reverse shear.

\subsection{Tension preload followed by shear}

Fig. 5 shows the experimental and predicted stress-strain response for 9 different cases: tensile preloads of 5, 10 and $20 \%$ along the $\mathrm{RD}$, each followed by shear reload at $45^{\circ}, 90^{\circ}$ and $135^{\circ}$ with respect to the RD.

The $135^{\circ}$ reload amounts practically to a reversal of the previous shear (associated with tension) in the 1-2 plane. More precisely the closeness between full strain reversal and the $135^{\circ}$ reload is characterized by the 'path change index' that equals- 1 for full reversal and that reaches a value of -0.92 in the present case (Raphanel et al., 1987). This leads to a plateau in the flow curve of Fig. 5 similar to the one obtained for reverse shear (Fig. 4). The $90^{\circ}$ reload amounts to 'cross-loading' and is the one with highest flow stress. The $45^{\circ}$ reload amounts, to some extent, to a continuation of the stress state associated 


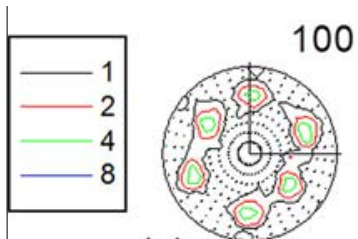

(a)

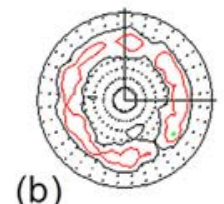

(b)
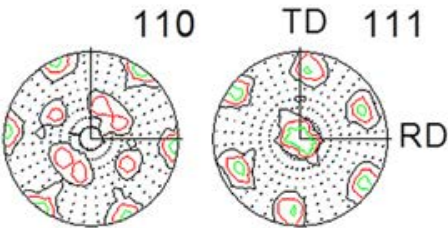

TD
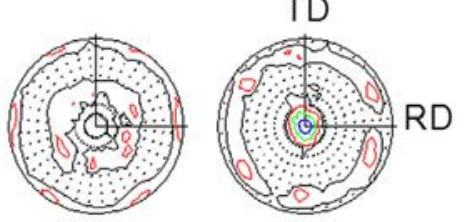

TD

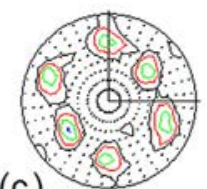

(c)
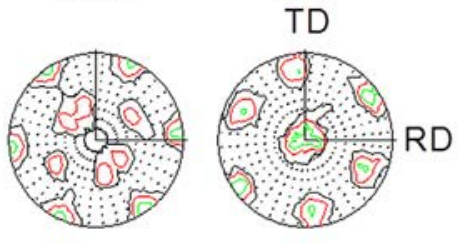
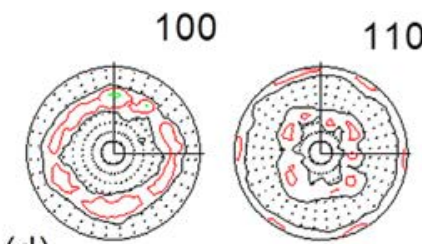

110

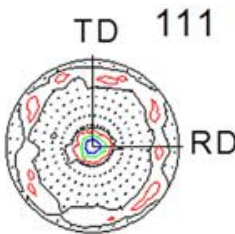

(d)
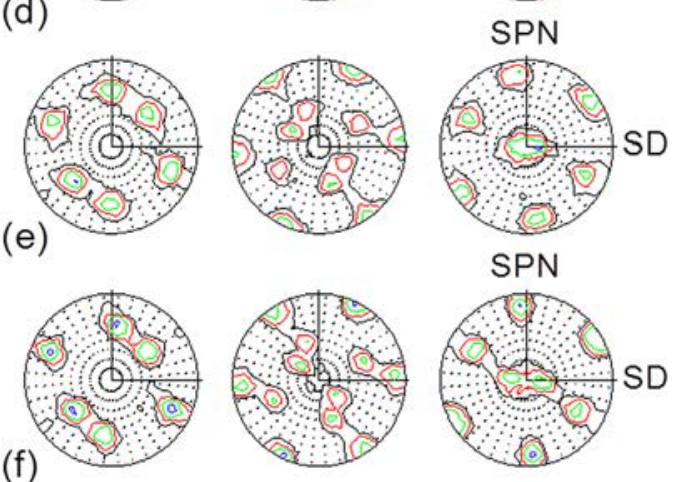

Fig. 9. Texture evolution after (a) 30\% forward shear followed by (b) 30\% reverse shear and (c) 60\% reverse shear; (d) 10\% RD tension followed by (e) 30\% shear at $90^{\circ}$ and (f) $60 \%$ shear at $90^{\circ}$. For (e) and (f) SD is shear direction and SPN shear plane normal.

with tension (the path change index is 0.92 ). The model slightly overpredicts the flow stress for the $45^{\circ}$ and $135^{\circ}$ reloads, but still captures the plateau in the $135^{\circ}$ reload associated with reversals, the relative ordering of the three tests, and the asymptotic hardening at large strain.

The evolution of dislocation densities (Fig. 7b) shows about the same evolution for the three cases. Essentially, because the 'reversibility parameter' $p$ is close to one until an accumulated strain of $40 \%$ (Fig. $6 \mathrm{~b}$ ), only reversible dislocations can be generated until then. The cases of $45^{\circ}$ and $90^{\circ}$ reloads involve little reversibility while for the $135^{\circ}$ shear test there is maximum reversibility and so dislocation recombination. As a consequence the associated reversible dislocation density remains smaller than the other two upon reload. Past $40 \%$ accumulated strain forward dislocations start to be generated.

The system activity associated with these strain path changes is very different from the one associated with shear/reverse shear (Fig $8 \mathrm{~b})$. The $\{112\}\langle 111\rangle$ systems are more active ( 60\%) during tension, but a discontinuous transition takes place at reload and the $\{110\}\langle 111\rangle$ systems contribute about $70 \%$ of the deformation, independently of the shear angle. Texture (Fig. 9d-f)) does not evolve substantially during the $10 \%$ tensile preload, but develops clear shear components after $30 \%$ and $60 \%$ reverse shear. We verified that suppressing texture evolution impacts not only the flow curve, as is to be expected, but also the evolution of forward and reversible dislocations.

\section{Conclusions}

We present here a crystallographic hardening model specifically developed for addressing strain path changes. The model is motivated by a previous continuum model (Rauch et al., 2011) with good predictive capability despite its simplicity. The continuum model is based on the observation that strain path changes tend to erase the previously formed dislocation structure independently of its details, and its predictive capabilities are quite good. The corollary is that one does not need to predict the details of the dislocation structure, but just get the dislocation densities right, at least for cubic materials. The main assumption of the model is that the dislocation population can be separated into reversible and forward (i.e., non-reversible) types, and that the former ones can be activated by reverse shear, so contributing to strain and being annihilated in the process. In the present model we follow the evolution of reversible and forward dislocations in each slip system of each grain. Accounting for grain orientation and crystallography allows us to account explicitly for several mechanism, not available to the continuum approach, such as: (1) the effect of texture evolution over the stress-strain response (geometric hardening) and the effect of grain rotation on switching on-off slip in specific systems; (2) there is no need to decide a priori what proportion of the dislocation population is to be converted into reversible or latent upon reload, since those categories follow from direction and amount of shear in each system.

The model relies on relatively few parameters, such as the reversibility, the latent hardening, the recombination, and the dislocation interaction strength. And we have, on purpose, assigned the same parameters to $\{110\}$ and $\{112\}$ slip in order to keep them to a minimum. An advantage of using a crystallographic framework is that the meaning and value of those parameters can be addressed with lower scale tools, such as Molecular Dynamics or Discrete Dislocation Dynamics. Another advantage of this formulation is that it provides virtual polycrystal responses needed for developing continuum constitutive models that account for deformation path changes during forming processes, such as the distortional hardening approach described in Barlat et al. (2011). In fact, continuum models with macroscopic constitutive descriptions are more convenient 
and time-efficient in numerical simulations of industrial forming processes in which loading is highly non-proportional. However, the identification of the relevant coefficients and the validation of the constitutive behavior are limited by the number and complexity of non-proportional loading experiments required for this purpose. Thus, the more physical hardening approach developed in the present work is particularly suitable to provide the virtual input data and the understanding necessary for the identification, validation and fine tuning of the macroscopic constitutive model.

\section{Acknowledgments}

CT acknowledges support from US Department of Energy, Office of Basic Energy Science, Division of Materials Science and Engineering, Project FWP 06SCPE401DOE-BES. JG, FB and KK, acknowledge support from Portuguese Foundation of Science and Technology (FCT) projects PEst-C/EME/UI0481/2011 and PTDC/EME-PME/116683/2010.

\section{Appendix A}

It has been pointed out by Hasegawa et al. (1986) and Takahashi and Shiono (1991) that forward and reverse stress-strain curves may be partly superimposed by shifting the curves on the strain axis. This is illustrated on Fig. A 1 for a $40 \%$ shear prestrain. The fraction $\mathrm{p}$ of dislocation converted into reversible segments is directly related to the shift $\Delta \gamma$ required to make the final parts of the curves coincident.
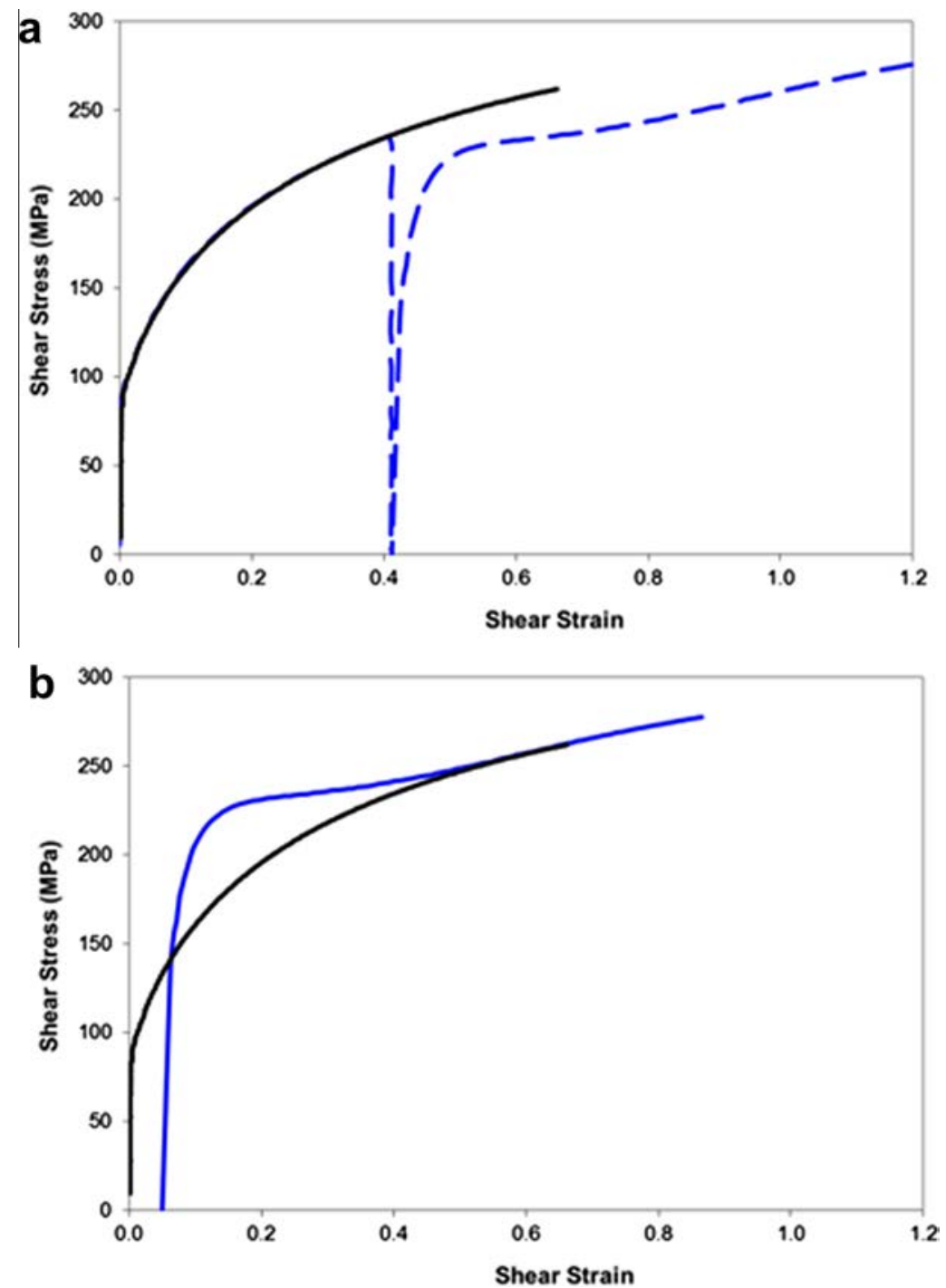

Fig. A1. Monotonic and reverse shear-stress data plotted (a) as measured and (b) shifted to make the final part of the curves coincident. 
Table A1

Fraction $p$ of dislocation converted into reversible segments obtained from measurements done in a low carbon steel subjected to increasing shear prestrains.

\begin{tabular}{lcccclll}
\hline Prestrain & 0.06 & 0.13 & 0.25 & 0.35 & 0.5 & 0.65 & 0.88 \\
$\Delta \gamma( \pm 0.02)$ & -0.02 & -0.01 & -0.01 & 0.02 & 0.08 & 0.21 & 0.5 \\
Parameter $p$ & 1 & 1 & 1 & 0.95 & 0.8 & 0.52 & 0.2 \\
\hline
\end{tabular}

If $\Delta \gamma$ equals the prestrain, then $p=0$. In such a case, strain is entirely cumulative or, in other words, there is no annihilation of previously stored defects. The surprising point is that $\Delta \gamma$ may vanish. This was observed to occur even for non-negligible prestrains (Rauch, 1991) and leads to $p=1$, which means that all the prestrain history is gradually 'erased' at reloading. More generally, because the parameter $p$ gives a measure of the dislocations that are permanently captured in the structure at reloading, an estimate may be obtained using the RGBV model. Indeed, the model gives a unique connection between dislocation density $\rho$ and shear strain $\rho=f(\gamma)$, with $f$ obtained by integrating numerically Eq. (3). The same relationship holds between the density of dislocation at reloading $\rho_{\text {for }}$ and the shift $\Delta \gamma$, i.e., following Eq. (4):

$$
\rho_{\text {for }}=(1-p) \rho_{\text {for }, 0}=f(\Delta \gamma)
$$

This equation, combined with the material parameters related to low carbon steel (Rauch et al., 2007), give the trend depicted in Table A1 and illustrated in Fig. A1a.

\section{References}

Bacroix, B., Brenner, R., 2012. A phenomenological anisotropic description for dislocation storage and recovery processes in fcc crystals. Computat. Mater. Sci. $54,97-100$.

Balint, D.S., Deshpande, V.S., Needleman, A., Van der Giessen, E., 2008. Discrete dislocation plasticity analysis of the grain size dependence of the flow strength of polycrystals. Int. J. Plasticity $24,2149-2172$.

Barlat, F., Gracio, J., Lee, M.G., Rauch, E.F., Vincze, G., 2011. An alternative to kinematic hardening in classical plasticity. Int. J. Plasticity 27, 1309-1327.

Beyerlein, I.J., Tomé, C.N., 2007. Modeling transients in the mechanical response of copper due to strain path changes. Int. J. Plasticity 23, 640-664.

Beyerlein, I.J., Tomé, C.N., 2008. A dislocation based constitutive law for pure Zr including temperature effects. Int. J. Plasticity 24, 867-895.

Brown, D.W., Beyerlein, I.J., Sisneros, T.A., Clausen, B., Tomé, C.N., 2012. Role of twinning and slip during compressive deformation of beryllium as a function of strain rate. Int. J. Plasticity $29,120-135$.

Capolungo, L., 2011. Dislocation junction formation and strength in magnesium. Acta Mater. 59, 2909-2917.

Cheong, K.S., Busso, E.P., Arsenlis, A., 2005. A study of microstructural length scale effects on the behaviour of FCC polycrystals using strain gradient concepts. Int. J. Plasticity 21, 1797-1814.

Devincre, B., Kubin, L., 2010. Scale transitions in crystal plasticity by dislocation dynamics simulations. Comptes Rendues Phys. 11, 274-284.

Franciosi, P., Berveiller, M., Zaoui, A., 1980. Latent hardening in copper and aluminium single crystals. Acta Metall. 28, $273-283$.

Hamelin, C.J., Diak, B.J., Pilkey, A.K., 2011. Multiscale modelling of the induced plastic anisotropy in bcc metals. Int. J. Plasticity 27, 1185-1202.

Hasegawa, T., Yakou, T., Kocks, U.F., 1986. Forward and reverse rearrangements of dislocations in tangled walls. Mater. Sci. Eng. A81, 189-199.

Kubin, L., Devincre, B., Hoc, T., 2008. Modeling dislocation storage rates and mean free paths in face-centered cubic crystals. Acta Mater. 56, 6040-6049.

Kysar, J.W., Saito, Y., Oztop, M.S., Lee, D., Huh, W.T., 2010. Experimental lower bounds on geometrically necessary dislocation density. Int. J. Plasticity 26, $1097-1123$.

Lebensohn, R.A., Tomé, C.N., 1993. A self-consistent anisotropic approach for the simulation of plastic deformation and texture development of polycrystals -Application to zirconium alloys. Acta Metall. Mater. 41, 2611-2624.

Lebensohn, R.A., Tomé, C.N., Ponte Castañeda, P., 2007. Self-consistent modeling of the mechanical behavior of viscoplastic polycrystals incorporating intragranular field fluctuations. Philos. Mag. 87, 4287-4322.

Lee, M.G., Lim, H., Adams, B.L., Hirth, J.P., Wagoner, R.H., 2010. A dislocation density-based single crystal constitutive equation. Int. J. Plasticity 26, 925-938.

Lopes, A.B., Barlat, F., Gracio, J.J., Ferreira Duarte, J.F., Rauch, E.F., 2003. Effect of texture and microstructure on strain hardening anisotropy for aluminum deformed in uniaxial tension and simple shear. Int. J. Plasticity 19, 1-22.

Madec, R., Kubin, L., 2008. Second-order junctions and strain hardening in bcc and fcc crystals. Scripta Mater. 58, 767-770.

M'Guil, S., Ahzi, S., Barlat, F., Gracio, J.J., 2011. Microstructural effects on yield surface evolution in cubic metals using the viscoplastic Phi-model. Int. J. Plasticity 27, 102-120.

Molinari, A., Canova, G.R., Ahzi, S., 1987. A self-consistent approach of the large deformation polycrystal viscoplasticity. Acta metall. 35, $2983-2994$.

Peeters, B., Kalidindi, S.R., Van Houtte, P., Aernoudt, E., 2000. A crystal plasticity based work hardening/softening model for BCC metals under changing strain paths. Acta Mater. 48, 2123-2133.

Peeters, B., Seefeldt, M., Teodosiu, C., Kalidindi, S.R., Van Houtte, P., Aernoudt, E., 2001a. Work-hardening/softening behaviour of BCC polycrystals during changing strain paths: I. An integrated model based on substructure and texture evolution, and its prediction of the stress-strain behaviour of an IF steel during two-stage strain paths. Acta Mater. 49, 1607-1619.

Peeters, B., Bacroix, B., Teodosiu, C., Van Houtte, P., Aernoudt, E., 2001b. Work-hardening/softening behaviour of BCC polycrystals during changing strain paths: II. TEM observations of dislocation sheets in an IF steel during two-stage strain paths and their representation in terms of dislocation densities. Acta Mater. 49, 1621-1632.

Proust, G., Tomé, C.N., Jain, A., Agnew, S.R., 2009. Modeling the effect of twinning and detwinning during strain-path changes of magnesium alloy AZ31. Int. J. Plasticity 25, 861-880.

Queyreau, S., Monnet, G., Devincre, B., 2009. Slip system interactions in a-iron determined by dislocation dynamic simulations. Int. J. Plasticity 25, 361-377.

Raphanel, J., Rauch, E., Shen, E.L., Schmitt, J.-H., 1987. Shear of prestrained steel specimens. Scripta Metall. 21, 1087-1090.

Rauch, E.F., 1991. Stress reversal tests imposed by shear on mild steel. In: Brandon, D.G. et al. (Eds.), Proceedings of ICSMA-9. Freund Publishing House, London, pp. 187-194.

Rauch, E.F., Gracio, J.J., Barlat, F., 2007. Work-hardening model for polycrystalline metals under strain reversal at large strain. Acta Mater. 55, $2939-2948$.

Rauch, E.F., Gracio, J.J., Barlat, F., Vincze, G., 2011. Modelling the plastic behavior of metals under complex loading conditions. Modell. Sim. Mater. Sci. Eng. 19 (035009), 1-18.

Rauch, E.F., Schmitt, J.H., 1989. Dislocation substructures in mild steel deformed in simple shear. Mater. Sci. Eng. A113, 441-448.

Rollett, A.D., Kocks, U.F., Doherty, R.D. 1987. Stage IV work hardening in cubic metals, formability and metallurgical structure, In: Dachdev, A.K., Embury, J.D. (Ed.), The Metallurgical Society, Warrendale, PA, pp. 211-225. 
Rossiter, J., Brahme, A., Simha, M.H., Inal, K., Mishra, R., 2010. A new crystal plasticity scheme for explicit time integration codes to simulate deformation in 3D microstructures: effects of strain path, strain rate and thermal softening on localized deformation in the aluminum alloy 5754 during simple shear. Int. J. Plasticity 26, 1702-1725.

Schmitt, J.-H., Shen, E.L., Raphanel, J.L., 1994. A parameter for measuring the magnitude of a change of strain path: validation and comparison with experiments on low carbon steel. Int. J. Plasticity 10, 535-551.

Segurado, J., Lebensohn, R.A., LLorca, J., Tomé, C.N., 2012. Multiscale modeling of plasticity based on embedding the viscoplastic self-consistent formulation in implicit finite elements. Int. J. Plasticity 28, 124-140.

Shiekhelsouk, M.N., Favier, V., Inal, K., Cherkaoui, M., 2009. Modelling the behaviour of polycrystalline austenitic steel with twinning-induced plasticity effect. Int. J. Plasticity 25, 105-133.

Tabourot, L., Fivel, M., Rauch, E.F., 1997. Generalised constitutive laws for fcc single crystals. Mater. Sci. Eng. A 234-236, 639-642.

Takahashi, H., Shiono, I., 1991. Backlash model for large deformation-behavior of aluminum under torsional cyclic loading. Int. J. Plasticity 7, $199-217$.

Vincze, G., Rauch, E.F., Gracio, J.J., Barlat, F., Lopes, A.B., 2005. A comparison of the mechanical behavior of an AA1050 and a low carbon steel deformed upon strain reversal. Acta Mater. 53, 1005-1013.

Yoon, J.W., Barlat, F., Gracio, J.J., Rauch, E., 2005. Anisotropic strain hardening behavior in simple shear for cube textured aluminum alloy sheets. Int. J. Plasticity 21, 2426-2447.

Weinberger, C.R., 2011. The structure and energetics of, and the plasticity caused by, Eshelby dislocations. Int. J. Plasticity $27,1391-1408$. 